National Institute on Drug Abuse

\title{
SEEKING DRUG ABUSE TREATMENT: KNOW WHAT TO ASK
}

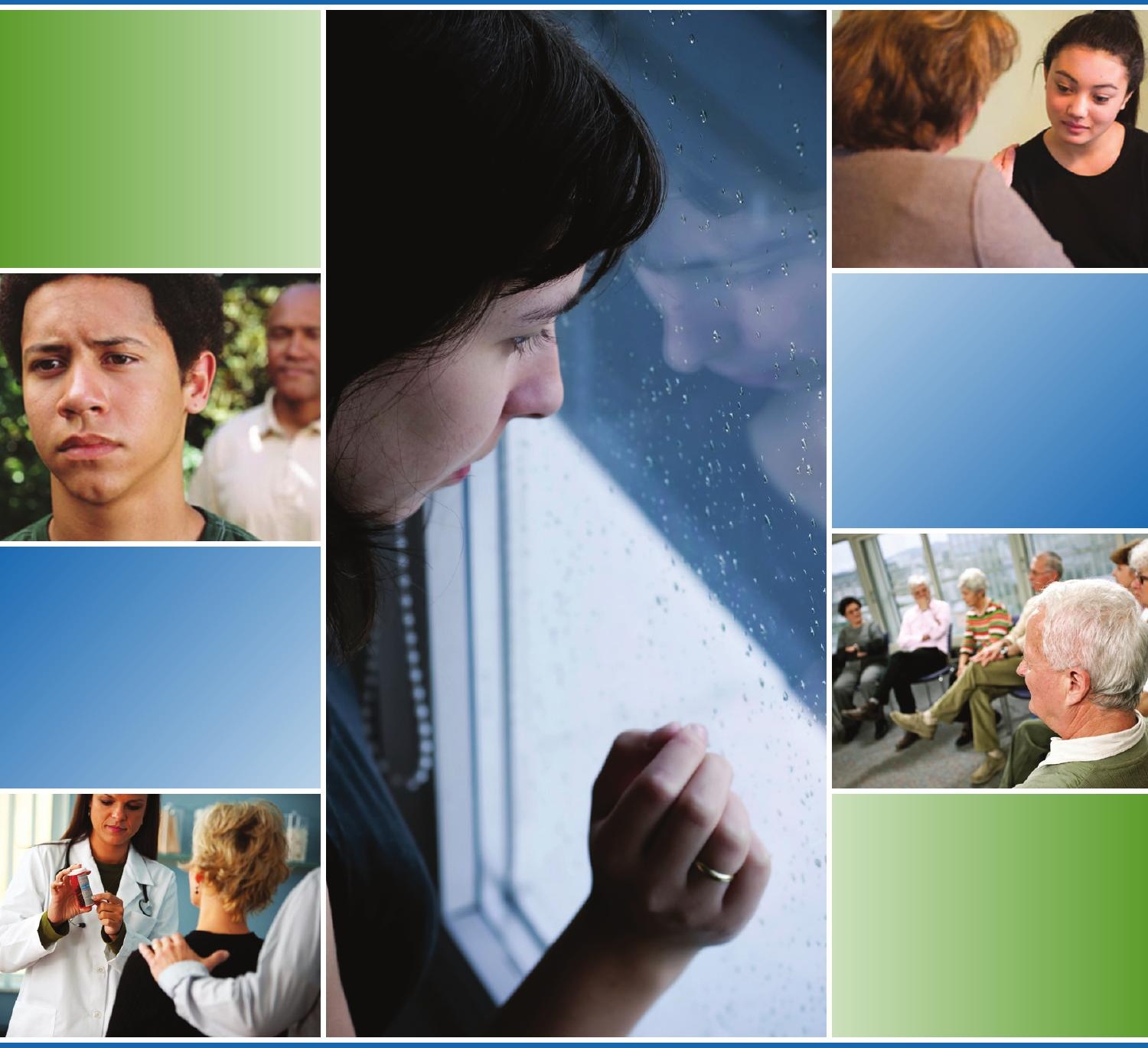

U.S. Department of Health and Human Services

National Institutes of Health 


\section{SEEKING DRUG ABUSE TREATMENT: KNOW WHAT TO ASK}

The goal of drug abuse treatment is to stop drug use and allow people to lead active lives in the family, workplace, and community. One continual challenge, however, is keeping patients in treatment long enough for them to achieve this goal.

That is why finding the right treatment for a person's specific needs is critical. Drug abuse treatment is not "one size fits all." Treatment outcomes depend on the:

- extent and nature of the person's problems;

- appropriateness of treatment;

- availability of additional services; and

- quality of interaction between the person and his or her treatment providers.

Family and friends can play important roles in motivating people with drug problems to enter and remain in treatment. However, trying to identify the right treatment programs for a loved one can be a difficult process.

To help, the National Institute on Drug Abuse (NIDA) created this brief guide containing five questions to ask when searching for a treatment program:

\section{Does the program use treatments backed by scientific evidence?}

\section{Effective drug abuse treatments can include behavioral therapy, medications, or, ideally, their combination.}

Behavioral therapies vary in focus and may involve:

- addressing a patient's motivation to change;

- providing incentives to stop taking drugs;

- building skills to resist drug use;

- replacing drug-using activities with constructive and rewarding activities;

- improving problem-solving skills; and

- building better personal relationships. 


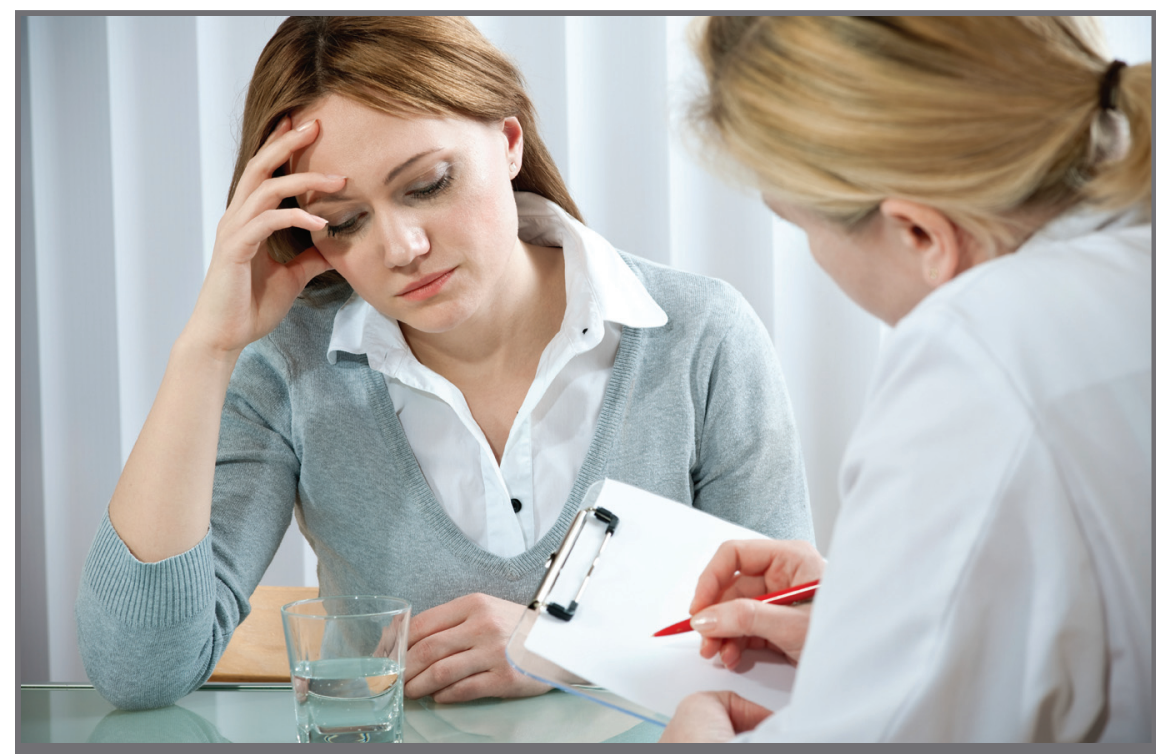

\section{Examples of Behavioral Therapies}

- Cognitive Behavioral Therapy. Seeks to help patients recognize, avoid, and cope with the situations in which they are most likely to abuse drugs.

- Motivational Incentives. Uses positive reinforcement such as providing rewards or privileges for remaining drug free, for participating in counseling sessions, or for taking treatment medications as prescribed.

- Motivational Interviewing. Uses strategies to encourage rapid and self-driven behavior change to stop drug use and help a patient enter treatment.

- Group Therapy. Helps patients face their drug abuse realistically, come to terms with its harmful consequences, and boost their motivation to stay drug free. Patients learn how to resolve their emotional and personal problems without abusing drugs.

Medications are an important part of treatment for many patients, especially when combined with counseling and other behavioral therapies. Different types of medications may be useful at different stages of treatment: to stop drug abuse, to stay in treatment, and to avoid relapse. 


\section{Components of Comprehensive Drug Abuse Treatment}

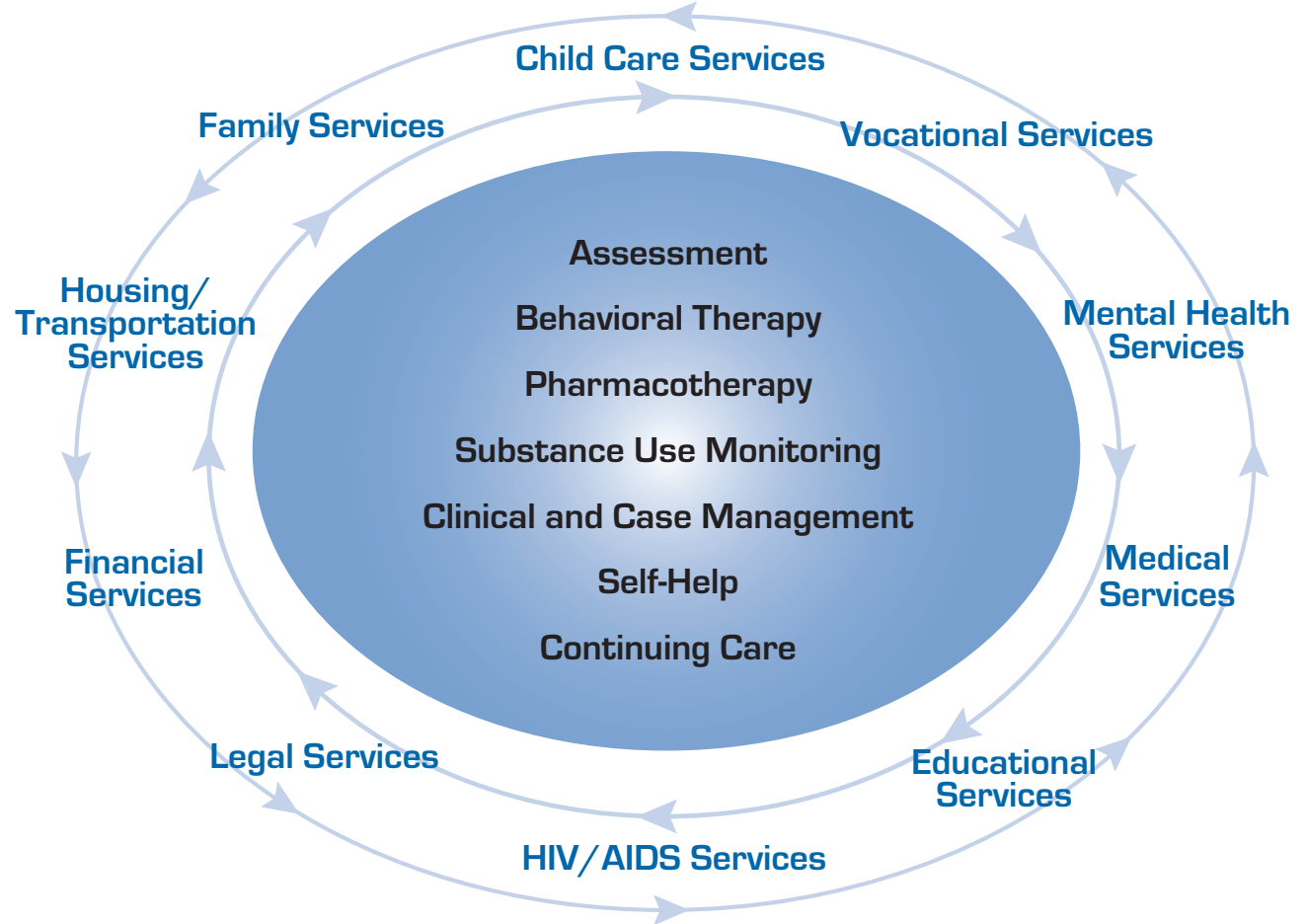

The best programs provide a combination of therapies and other services to meet a patient's needs.

3. Does the program adapt treatment as the patient's needs change?

\section{Individual treatment and service plans must be assessed and modified as needed to meet changing needs.}

A person in treatment may require varying combinations of services during its course, including ongoing assessment. For instance, the program should build in drug monitoring so the treatment plan can be adjusted if relapse occurs. For most people, a continuing care approach provides the best results, with treatment level adapted to a person's changing needs.

A patient's needs for support services, such as day care or transportation, should also be met during treatment. 


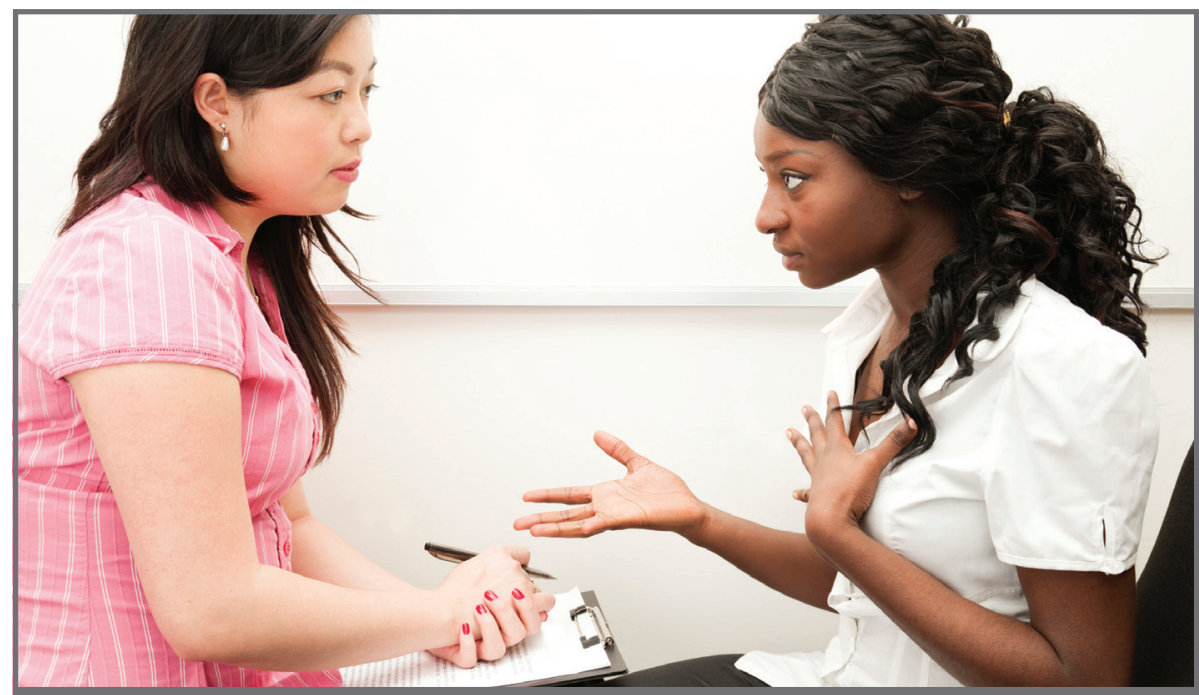

\section{Relapse Does Not Mean Treatment Failure}

The chronic nature of addiction means that relapsing to drug abuse is not only possible, but likely, similar to what happens with other chronic medical illnesses-such as diabetes, hypertension, and asthma-that have both physical and behavioral components. And like these illnesses, addiction also requires continual evaluation and treatment modification if necessary.

\section{Is the duration of treatment sufficient?}

Remaining in treatment for the right period of time is critical.

Appropriate time in treatment depends on the type and degree of a person's problems and needs. Research tells us that most addicted people need at least three months in treatment to really reduce or stop their drug use and that longer treatment times result in better outcomes. The best programs will measure progress and suggest plans for maintaining recovery. Recovery from drug addiction is a long-term process that often requires several episodes of treatment and ongoing support from family or community.

\section{A relapse to drug use indicates a need to re-instate or adjust treatment strategy; it does not mean treatment has failed.}




\section{How do 12-step or similar recovery programs fit into drug addiction treatment?}

\section{Self-help groups can complement and extend the effects of professional treatment.}

The most well-known programs are Alcoholics Anonymous (AA), Narcotics Anonymous (NA), and Cocaine Anonymous (CA), all of which are based on the 12-step model. This group therapy model draws on the social support offered by peer discussion to help promote and sustain drug-free lifestyles.

Most drug addiction treatment programs encourage patients to participate in group therapy during and after formal treatment. These groups offer an added layer of community-level social support to help people in recovery with abstinence and other healthy lifestyle goals.

\section{Additional Treatment Resources}

- For science-based principles of treatment see NIDA's Principles of Drug Addiction Treatment: A Research Based Guide at http://www.drugabuse.gov/PODAT/PODATIndex.html.

- Substance Abuse and Mental Health Services Administration (SAMHSA) Treatment Locator: www.findtreatment.samhsa.gov; 1-800-662-HELP.

- The "Find a Physician" feature on the American Society of Addiction Medicine (ASAM) Web site: http://www.asam.org/for-the-public/treatment.

- The Patient Referral Program on the American Academy of Addiction Psychiatry Web site: http://www.aaap.org/patient-referral-program.

- The Child and Adolescent Psychiatrist Finder on the American Academy of Child \& Adolescent Psychiatry Web site: http://www.aacap.org/cs/root/child_and_adolescent_psychiatrist_ finder/child_and_adolescent_psychiatrist_finder.

- For clinical trials information, go to www.clinicaltrials.gov.

\section{Other Useful Links}

- National Suicide Prevention Lifeline: 1-800-273-TALK.

- National Institute on Drug Abuse (NIDA): www.drugabuse.gov.

- NIDA for Teens: www.teens.drugabuse.gov.

- NIDA’s Easy-to-Read Drug Facts: www.easyread.drugabuse.gov.

- National Institute on Alcohol Abuse and Alcoholism: www.niaaa.nih.gov.

- National Institute of Mental Health: www.nimh.nih.gov.

- Faces and Voices of Recovery: www.facesandvoicesofrecovery.org.

- The Partnership at DrugFree.org: www.drugfree.org.

To order NIDA materials, please go to: http://drugpubs.drugabuse.gov. 
NH National Institute on Drug Abuse

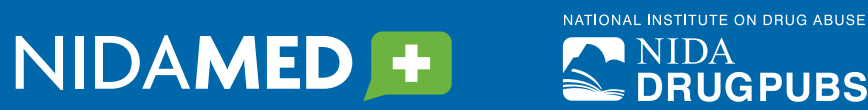

U.S. Department of Health and Human Services

This publication is in the public domain and may be used or reproduced in its entirety without permission from NIDA. Citation of the source is appreciated. 